SHORT REPORT

\title{
Extravasation injuries on regional neonatal units
}

\author{
C E Wilkins, A J B Emmerson
}

Arch Dis Child Fetal Neonatal Ed 2004;89:F274-F275. doi: 10.1136/adc.2003.028241

This survey of regional neonatal intensive care units determined a prevalence of 38 per 1000 neonates who sustained an extravasation injury that caused skin necrosis. Most injuries occurred in infants of 26 weeks gestation or less, with parenteral nutrition infused through intravenous cannulae. Common treatments were exposing wounds to the air, infiltration with hyaluronidase and saline, and occlusive dressings.

$\mathrm{E}$ xtravasation is the non-intentional leakage of infused fluid into surrounding tissue, which may cause damage. Extravasation or infiltration of fluid has been shown to occur in up to $70 \%$ of neonates, although tissue damage and skin necrosis is much less common. ${ }^{1}$ About $4 \%$ of infants leave neonatal intensive care units with cosmetically or functionally significant scars, thought to be caused by extravasation injuries. ${ }^{2}$ Certainly very preterm infants who are dependent on intravenous infusions for their survival are vulnerable to this type of injury.

At present, there is no agreement on the best practice for managing these injuries. In the literature ${ }^{13}$ and textbooks, ${ }^{4}$ a number of treatments are advocated, including hyaluronidase and saline infiltrations, hydrocolloids, and hydrogels. In this study, we established the prevalence of extravasation injuries that cause skin necrosis in neonatal intensive care unit populations and how these injuries were managed on different units in the United Kingdom in 2002.

\section{METHOD}

Two copies of a questionnaire were sent to the 38 regional neonatal intensive care units in the United Kingdom, one to a consultant and one to a senior nurse. Respondents were asked to identify any baby on the unit, at that time, who had sustained an extravasation injury causing skin necrosis (fig 1), and to give information about the type of line and infusion that caused the injury and its subsequent management. Details on the unit were also requested.

\section{RESULTS}

Replies were received from 31 units (82\% of those surveyed). From 13 units, two questionnaires were returned; the reply giving the most detailed information was used in the analysis. At the time of the survey, there were 742 babies on the units, the total unit capacity was 852, with 308 intensive care cots and 255 high dependency cots.

There were 28 injuries reported, which gives a prevalence of 38 per 1000 babies sustaining an extravasation injury causing skin necrosis. The mean gestation of these babies was 26.1 weeks (range 23-35). Most of the injuries, 26 (93\%), were from peripheral intravenous cannulae, with two (7\%) caused by peripherally inserted central lines. Parenteral nutrition caused more injuries than any other infusion (14/ 28); $10 \%$ dextrose+calcium and blood both caused 5/28 injuries. Other infusions implicated were sodium bicarbonate (2/28), Tham (2/28), 10\% dextrose+flucloxacillin (1/28), insulin+aminophylline $(1 / 28), \quad 10 \%$ dextrose+magnesium $(1 / 28)$, and platelets $(1 / 28)$. In four cases, two infusions were running simultaneously.

A variety of treatments were given. Exposing the wound to the air was used in 12 cases (43\%): in eight cases alone and four with other treatments. Twelve (43\%) wounds were treated by infiltration, 10 of these with hyaluronidase and normal saline, and two with normal saline alone. Occlusive dressings were used in 12 cases: nine $(20 \%)$ used hydrocolloids, two $(6.5 \%)$ used hydrogels, and one was treated with a film dressing. In eight cases, two treatments were identified.

In half of the cases, neonatal unit staff were identified as being solely responsible for care of the wound. Ten (36\%) cases were referred to plastic surgeons; of these, seven were treated by infiltration, two with hydrocolloids, and one was exposed to the air. In only five cases were specialist wound care nurses involved.

\section{DISCUSSION}

The prevalence of 38 per 1000 infants sustaining an extravasation injury that resulted in skin necrosis is relatively low considering that only regional neonatal units were surveyed. Over a third of the cots were intensive care cots, and it could be expected that these units would be looking after a high proportion of the sickest preterm infants.

Most injuries (70\%) occurred in infants of 26 weeks gestation and less. These very preterm infants have the most immature skin, which is easily damaged. They often require a longer duration of intravenous therapy, and obtaining intravenous access can be difficult.

Most injuries occurred with intravenous cannulae. Parenteral nutrition was the infusion most often implicated as the cause of the injury. The data suggest that the prolonged use of parenteral nutrition through intravenous cannulae should be avoided, whenever possible, particularly in very immature infants.

This survey has confirmed a lack of standardised treatment for extravasation injuries. Leaving the wound exposed, infiltration with hyaluronidase and saline, and occlusive dressings were used with equal frequency. Currently, leaving the wound exposed to the air is not considered the optimal way to treat these injuries. ${ }^{1}$ The dry scab that covers the wound is thought to delay epithelisation. Occlusive dressings have the advantage of keeping the wound moist, which aids healing. Infiltration with hyaluronidase and saline is an invasive procedure recommended in standard texts, ${ }^{4}$ and there are case reports showing its use. ${ }^{3}$ However, there have been no studies in preterm infants comparing its effectiveness with other treatments. In addition the British National Formulary recommends hyaluronidase to be used with caution in infants.

\section{Conclusions}

There is no consensus on the management of extravasation injuries in preterm infants. For this population, no clinical trials have been performed that compare the outcome of treatment with occlusive dressings with that of infiltration with hyaluronidase and saline, even though this study 


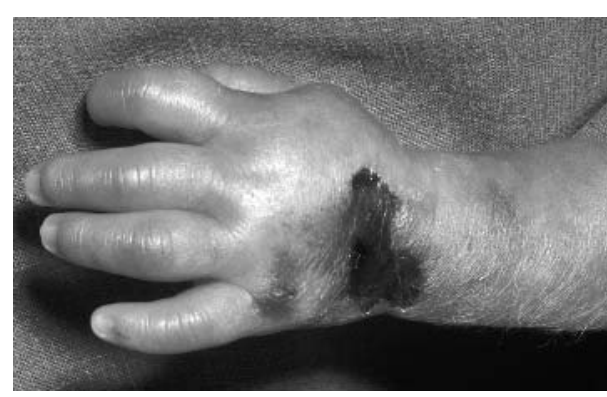

Figure 1 Intravenous drip lesion.

confirms that both treatments are commonly used in the United Kingdom. Further research is needed to help prevent these injuries, and to determine which is the best treatment to aid healing and reduce scaring.

\section{Authors' affiliations}

C Wilkins, A Emmerson, Neonatal Medical Unit, St Mary's Hospital, Whitworth Park, Manchester M13 OJH, UK

Correspondence to: Dr Wilkins, Neonatal Medical Unit, St Mary's Hospital, Whitworth Park, Manchester M13 0JH, UK; clare.wilkins@ lineone.net

Accepted 13 May 2003

\section{REFERENCES}

1 Irving V. Neonatal iatrogenic skin injuries: a nursing perspective. Journal of Neonatal Nursing 1999;5:10-13.

2 Cartildge PHT, Fox PE, Rutter N. The scars of newborn intensive care. Early Hum Dev 1990;21:1-10.

3 Davies J, Gault D, Buchdahl R. Preventing the scars of neonatal intensive care. Arch Dis Child Fetal Neonatal Ed 1994;70:F50-1.

4 Rennie J, Roberton N, eds. Textbook of neonatology, 3rd ed. Edinburgh: Churchill Livingstone, 1999.

5 British National Formulary. London: BMJ Books, 2002;44. 